\title{
REAL HYPERSURFACES IN COMPLEX TWO-PLANE GRASSMANNIANS WITH PARALLEL SHAPE OPERATOR
}

\author{
YOUNG JIN SUH
}

\begin{abstract}
In this paper we show that there do not exist any real hypersurfaces in a complex two dimensional Grassmannians $G_{2}\left(\mathbb{C}^{n+2}\right)$ with parallel shape operator.
\end{abstract}

\section{INTRODUCTION}

In the geometry of real hypersurfaces in complex space form $M_{n}(c)$ or in quaternionic space forms it can be easily checked that there do not exist any real hypersurfaces with parallel shape operator $A$ by virture of the equation of Codazzi.

From this point of a view many differential geometers have considered a much more weaker notion such as a parallel second fundamental form, that is, $\nabla A=0$. In particular, Kimura and Maeda [5] have proved that a real hypersurface $M$ in a complex projective space $C P^{m}$ satisfying $\nabla_{\xi} A=0$ is locally congruent to a real hypersurface of type $A_{1}, A_{2}$, that is, a tube over a totally geodesic complex submanifold $C P^{k}$. with radius $0<r<\pi / 2$. The structure vector field $\xi$ mentioned above is defined by $\xi=-J N$, where $J$ denotes a Kähler structure of $C P^{m}$ and $N$ a local unit normal field of $M$ in $C P^{m}$. Moreover, in a class of Hopf hypersurfaces Kimura [4] has asserted that there do not exist any real hypersurfaces with parallel Ricci tensor, that is $\nabla S=0$, where $S$ denotes the Ricci tensor of a real hypersurface $M$ in $C P^{m}$.

On the other hand, in a quaternionic projective space $Q P^{m}$ Pérez [6] has considered the notion of $\nabla_{\xi_{i}} A=0, i=1,2,3$, for real hypersurfaces in $Q P^{m}$ and proved that $M$ is locally congruent to a hypersurface of $A_{1}, A_{2}$-type, that is, a tube over $Q P^{k}$ with radius $0<r<\pi / 4$. The almost contact structure vector fields $\left\{\xi_{1}, \xi_{2}, \xi_{3}\right\}$ are defined by $\xi_{i}=-J_{i} N, i=1,2,3$, where $J_{i}$ denotes a quaternionic Kähler structure of $Q P^{m}$ and $N$ a unit normal field of $M$ in $Q P^{m}$. Moreover, Pérez and the present author [7] have considered the notion of $\nabla_{\xi_{i}} R=0, i=1,2,3$, where $R$ denotes the curvature tensor of a real hypersurface $M$ in $Q P^{m}$, and proved that $M$ is locally congruent to a tube of radius $\pi / 4$ over $Q P^{k}$.

Received 13th January, 2003

This work was supported by grant Proj. No R05-2002-000-00047-0 from the Basic Research Program of the Korea Science \& Engineering Foundation.

Copyright Clearance Centre, Inc. Serial-fee code: 0004-9727/03 \$A2.00+0.00. 
Now let us denote by $G_{2}\left(\mathbb{C}^{m+2}\right)$ the set of all complex 2-dimensional linear subspace of $\mathbb{C}^{m+2}$. Then the situation mentioned above is not so simple if we consider a real hypersurface in such a 2-dimensional complex Grassmannian $G_{2}\left(\mathbb{C}^{m+2}\right)$.

In this paper we study the analogous question in the complex Grassmann manifold $G_{2}\left(\mathbb{C}^{m+2}\right)$ of all two-dimensional linear subspaces in $\mathbb{C}^{m+2}$. This Riemannian symmetric space has a remarkable geometrical structure. It is the unique compact irreducible Riemannian manifold being equipped with both a Kähler structure $J$ and a quaternionic Kähler structure $\mathfrak{J}$ not containing $J$. In other words, $G_{2}\left(\mathbb{C}^{m+2}\right)$ is the unique compact, irreducible, Kähler, quaternionic Kähler manifold which is not a hyperkähler manifold. So, in $G_{2}\left(\mathbb{C}^{m+2}\right)$ we have the two natural geometrical conditions for real hypersurfaces that $[\xi]=\operatorname{Span}\{\xi\}$ or $\mathfrak{D}^{\perp}=\operatorname{Span}\left\{\xi_{1}, \xi_{2}, \xi_{3}\right\}$ is invariant under the shape operator. From such a view point Berndt and the present author [2] have proved the following.

TheOREM A. Let $M$ be a connected real hypersurface in $G_{2}\left(\mathbb{C}^{m+2}\right), m \geqslant 3$. Then both $[\xi]$ and $\mathfrak{D}^{\mathcal{L}}$ are invariant under the shape operator of $M$ if and only if

(1) $M$ is an open part of a tube around a totally geodesic $G_{2}\left(\mathbb{C}^{m+1}\right)$ in $G_{2}\left(\mathbb{C}^{m+2}\right)$, or

(2) $m$ is even, say $m=2 n$, and $M$ is an open part of a tube around a totally geodesic $\mathbb{H} P^{n}$ in $G_{2}\left(\mathbb{C}^{m+2}\right)$.

In the proof of Theorem A we have proved that the one-dimensional distribution $[\xi]$ is contained in either the 3-dimensional distribution $\mathfrak{D}^{\perp}$ or in the orthogonal complement $\mathfrak{D}$ such that $T_{x} M=\mathfrak{D} \oplus \mathfrak{D}^{\perp}$. The case (2) in Theorem A is just the case that the one dimensional distribution $[\xi]$ is contained in $\mathfrak{D}^{\perp}$. Of course it is not difficult to check that the second fundamental form of any real hypersurfaces given in Theorem $\mathrm{A}$ is not parallel. Then it must be natural question to know that whether real hypersurfaces in $G_{2}\left(\mathbb{C}^{m+2}\right)$ whose second fundamental form is parallel can exist or not?

The main result of this paper is to prove the non-existence of all real hypersurfaces in $G_{2}\left(\mathbb{C}^{m+2}\right)$ with parallel second fundamental tensor in the following theorem.

THEOREM. There do not exist any real hypersurface $M$ in $G_{2}\left(\mathbb{C}^{m+2}\right)$ with parallel second fundamental form, that is, $\nabla A=0$.

In Section 2 we recall the Riemannian geometry of two dimensional complex Grassmannian $G_{2}\left(\mathbb{C}^{m+2}\right)$, and in Section 3 we shall show some fundamental properties of real hypersurfaces in $G_{2}\left(\mathbb{C}^{m+2}\right)$. The equation of Codazzi will be proved explicitly in this section. In Section 4 we will give a complete proof of the main Theorem given in the introduction.

The proof of our main Theorem also works for the more general case of Codazzi hypersurfaces, that is, for hypersurfaces whose shape operator is a Codazzi tensor, 
which means that the covariant derivative of the shape operator is symmetric in all its variables.

\section{RIEMANNIAN GEOMETRY OF $G_{2}\left(\mathbb{C}^{m+2}\right)$}

In this section we summarise basic material about $G_{2}\left(\mathbb{C}^{m+2}\right)$, for details we refer to $[1,2,3]$. By $G_{2}\left(\mathbb{C}^{m+2}\right)$ we denote the set of all complex two-dimensional linear subspaces in $\mathbb{C}^{m+2}$. The special unitary group $G=S U(m+2)$ acts transitively on $G_{2}\left(\mathbb{C}^{m+2}\right)$ with stabiliser isomorphic to $K=S(U(2) \times U(m)) \subset G$. Then $G_{2}\left(\mathbb{C}^{m+2}\right)$ can be identified with the homogeneous space $G / K$, which we equip with the unique analytic structure for which the natural action of $G$ on $G_{2}\left(\mathbb{C}^{m+2}\right)$ becomes analytic. Denote by $\mathfrak{g}$ and $\mathfrak{k}$ the Lie algebra of $G$ and $K$, respectively, and by $\mathfrak{m}$ the orthogonal complement of $\mathfrak{k}$ in $\mathfrak{g}$ with respect to the Cartan-Killing form $B$ of $\mathfrak{g}$. Then $\mathfrak{g}=\mathfrak{k} \oplus \mathfrak{m}$ is an $A d(K)$-invariant reductive decomposition of $\mathfrak{g}$. We put $o=e K$ and identify $T_{o} G_{2}\left(\mathbb{C}^{m+2}\right)$ with $\mathfrak{m}$ in the usual manner. Since $B$ is negative definite on $\mathfrak{g}$, its negative restricted to $\mathfrak{m} \times \mathfrak{m}$ yields a positive definite inner product on m. By $A d(K)$-invariance of $B$ this inner product can be extended to a $G$-invariant Riemannian metric $g$ on $G_{2}\left(\mathbb{C}^{m+2}\right)$. In this way $G_{2}\left(\mathbb{C}^{m+2}\right)$ becomes a Riemannian homogeneous space, even a Riemannian symmetric space. For computational reasons we normalise $g$ such that the maximal sectional curvature of $\left(G_{2}\left(\mathbb{C}^{m+2}\right), g\right)$ is eight. Since $G_{2}\left(\mathbb{C}^{3}\right)$ is isometric to the three-dimensional complex projective space $\mathbb{C} P^{3}$ with constant holomorphic sectional curvature eight we shall assume $m \geqslant 2$ from now on. Note that the isomorphism $\operatorname{Spin}(6) \simeq S U(4)$ yields an isometry between $G_{2}\left(\mathbb{C}^{4}\right)$ and the real Grassmann manifold $G_{2}^{+}\left(\mathbb{R}^{6}\right)$ of oriented two-dimensional linear subspaces of $\mathbb{R}^{6}$.

The Lie algebra $\mathfrak{k}$ has the direct sum decomposition $\mathfrak{k}=\mathfrak{s u}(m) \oplus \mathfrak{s} u(2) \oplus \Re$, where $\mathfrak{R}$ is the centre of $\mathfrak{k}$. Viewing $\mathfrak{k}$ as the holonomy algebra of $G_{2}\left(\mathbb{C}^{m+2}\right)$, the centre $\mathfrak{R}$ induces a Kähler structure $J$ and the $\mathfrak{s} u(2)$-part a quaternionic Kähler structure $\mathfrak{J}$ on $G_{2}\left(\mathbb{C}^{m+2}\right)$. If $J_{1}$ is any almost Hermitian structure in $\mathfrak{J}$, then $J J_{1}=J_{1} J$, and $J J_{1}$ is a symmetric endomorphism with $\left(J J_{1}\right)^{2}=I$ and $\operatorname{tr}\left(J J_{1}\right)=0$. This fact will be used frequently throughout this paper.

A canonical local basis $J_{1}, J_{2}, J_{3}$ of $\mathfrak{J}$ consists of three local almost Hermitian structures $J_{\nu}$ in $\mathfrak{J}$ such that $J_{\nu} J_{\nu+1}=J_{\nu+2}=-J_{\nu+1} J_{\nu}$, where the index is taken module three. Since $\mathfrak{J}$ is parallel with respect to the Riemannian connection $\bar{\nabla}$ of $\left(G_{2}\left(\mathbb{C}^{m+2}\right), g\right)$, there exist for any canonical local basis $J_{1}, J_{2}, J_{3}$ of $\mathfrak{J}$ three local one-forms $q_{1}, q_{2}, q_{3}$ such that

$$
\bar{\nabla}_{X} J_{\nu}=q_{\nu+2}(X) J_{\nu+1}-q_{\nu+1}(X) J_{\nu+2}
$$

for all vector fields $X$ on $G_{2}\left(\mathbb{C}^{m+2}\right)$. Also this fact will be used frequently. 
Let $p \in G_{2}\left(\mathbb{C}^{m+2}\right)$ and $W$ a subspace of $T_{p} G_{2}\left(\mathbb{C}^{m+2}\right)$. We say that $W$ is a quaternionic subspace of $T_{p} G_{2}\left(\mathbb{C}^{m+2}\right)$ if $J W \subset W$ for all $J \in \mathfrak{J}_{p}$. And we say that $W$ is a totally complex subspace of $T_{p} G_{2}\left(\mathbb{C}^{m+2}\right)$ if there exists a one-dimensional subspace $\mathfrak{V}$ of $\mathfrak{J}_{p}$ such that $J W \subset W$ for all $J \in \mathfrak{V}$ and $J W \perp W$ for all $J \in \mathfrak{V}^{\perp} \subset \mathfrak{J}_{p}$. Here, the orthogonal complement of $\mathfrak{V}$ in $\mathfrak{J}_{p}$ is taken with respect to the bundle metric and orientation on $\mathfrak{J}$ for which any local oriented orthonormal frame field of $\mathfrak{J}$ is a canonical local basis of $\mathfrak{J}$. A quaternionic (respectively, totally complex) submanifold of $G_{2}\left(\mathbb{C}^{m+2}\right)$ is a submanifold all of whose tangent spaces are quaternionic (respectively, totally complex) subspaces of the corresponding tangent spaces of $G_{2}\left(\mathbb{C}^{m+2}\right)$.

The Riemannian curvature tensor $\bar{R}$ of $G_{2}\left(\mathbb{C}^{m+2}\right)$ is locally given by

$$
\begin{gathered}
\bar{R}(X, Y) Z=g(Y, Z) X-g(X, Z) Y+g(J Y, Z) J X-g(J X, Z) J Y-2 g(J X, Y) J Z \\
+\sum_{\nu=1}^{3}\left\{g\left(J_{\nu} Y, Z\right) J_{\nu} X-g\left(J_{\nu} X, Z\right) J_{\nu} Y-2 g\left(J_{\nu} X, Y\right) J_{\nu} Z\right\} \\
+\sum_{\nu=1}^{3}\left\{g\left(J_{\nu} J Y, Z\right) J_{\nu} J X-g\left(J_{\nu} J X, Z\right) J_{\nu} J Y\right\}
\end{gathered}
$$

where $J_{1}, J_{2}, J_{3}$ is any canonical local basis of $\mathfrak{J}$.

\section{REAL HYPERSURFACES IN $G_{2}\left(\mathbb{C}^{m+2}\right)$}

Let $M$ be a real hypersurface of $G_{2}\left(\mathbb{C}^{m+2}\right)$, that is, a hypersurface of $G_{2}\left(\mathbb{C}^{m+2}\right)$ with real codimension one. The induced Riemannian metric on $M$ will also be denoted by $g$, and $\nabla$ denotes the Riemannian connection of $(M, g)$. We denote by $\mathfrak{H}$ and $\mathfrak{D}$ the maximal complex and quaternionic subbundle of the tangent bundle $T M$ of $M$, respectively. Let $N$ be a local unit normal field of $M$ and $A$ the shape operator of $M$ with respect to $N$. The Kähler structure $J$ of $G_{2}\left(\mathbb{C}^{m+2}\right)$ induces on $M$ a local almost contact metric structure $(\phi, \xi, \eta, g)$, where $\phi X$ is the tangential component of $J X, \xi=-J N$, and $\eta(X)=g(X, \xi)$. Furthermore, let $J_{1}, J_{2}, J_{3}$ be a canonical local basis of $\mathfrak{J}$. Then each $J_{\nu}$ induces a local almost contact metric structure $\left(\phi_{\nu}, \xi_{\nu}, \eta_{\nu}, g\right)$ on $M$. Locally, $\mathfrak{H}$ is the orthogonal complement in $T M$ of the span of $\xi$, and $\mathfrak{D}$ is the orthogonal complement of $\mathfrak{D}^{\perp}=\operatorname{Span}\left\{\xi_{1}, \xi_{2}, \xi_{3}\right\}$ in $T M$.

We shall now derive some basic equations involving the elements in these almost contact metric structures. First of all, from the identity $J^{2}=-I$ we get for all $X \in T M$

$$
-X=J^{2} X=J(\phi X+\eta(X) N)=J \phi X-\eta(X) \xi=\phi^{2} X+\eta(\phi X) N-\eta(X) \xi .
$$

The tangential and normal component of this equation imply 
LEMMA 3.1. For all $X \in T M$ we have

$$
\phi^{2} X=-X+\eta(X) \xi \text { and } \eta(\phi X)=0 .
$$

Next, we have

$$
\begin{aligned}
J J_{\nu} X & =J\left(\phi_{\nu} X+\eta_{\nu}(X) N\right)=J \phi_{\nu} X-\eta_{\nu}(X) \xi \\
& =\phi \phi_{\nu} X+\eta\left(\phi_{\nu} X\right) N-\eta_{\nu}(X) \xi
\end{aligned}
$$

and

$$
\begin{aligned}
J_{\nu} J X & =J_{\nu}(\phi X+\eta(X) N)=J_{\nu} \phi X-\eta(X) \xi_{\nu} \\
& =\phi_{\nu} \phi X+\eta_{\nu}(\phi X) N-\eta(X) \xi_{\nu}
\end{aligned}
$$

The tangential and normal component of the identity $J J_{\nu}=J_{\nu} J$ therefore gives

LEMMA 3.2. For all $X \in T M$ we have

$$
\phi \phi_{\nu} X-\phi_{\nu} \phi X=\eta_{\nu}(X) \xi-\eta(X) \xi_{\nu} \text { and } \eta_{\nu}(\phi X)=\eta\left(\phi_{\nu} X\right)
$$

From the second equation in Lemma 3.2 we get

$$
g\left(\phi_{\nu} \xi, X\right)=-g\left(\xi, \phi_{\nu} X\right)=-\eta\left(\phi_{\nu} X\right)=-\eta_{\nu}(\phi X)=-g\left(\xi_{\nu}, \phi X\right)=g\left(\phi \xi_{\nu}, X\right)
$$

and hence we assert

Corollary 3.1 .

$$
\phi_{\nu} \xi=\phi \xi_{\nu}
$$

Next, we have

$$
\begin{aligned}
J_{\nu} J_{\nu+1} X & =J_{\nu}\left(\phi_{\nu+1} X+\eta_{\nu+1}(X) N\right) \\
& =\phi_{\nu} \phi_{\nu+1} X+\eta_{\nu}\left(\phi_{\nu+1} X\right) N-\eta_{\nu+1}(X) \xi_{\nu}
\end{aligned}
$$

and

$$
\begin{aligned}
-J_{\nu+1} J_{\nu} X & =-J_{\nu+1}\left(\phi_{\nu} X+\eta_{\nu}(X) N\right) \\
& =-\phi_{\nu+1} \phi_{\nu} X-\eta_{\nu+1}\left(\phi_{\nu} X\right) N+\eta_{\nu}(X) \xi_{\nu+1}
\end{aligned}
$$

Since

$$
J_{\nu+2} X=\phi_{\nu+2} X+\eta_{\nu+2}(X) N
$$

the tangential and normal components of

$$
J_{\nu} J_{\nu+1}=J_{\nu+2}=-J_{\nu+1} J_{\nu}
$$

imply the following 
LEMMA 3.3. For all $X \in T M$ we have

$$
\phi_{\nu} \phi_{\nu+1} X-\eta_{\nu+1}(X) \xi_{\nu}=\phi_{\nu+2} X=-\phi_{\nu+1} \phi_{\nu} X+\eta_{\nu}(X) \xi_{\nu+1}
$$

and

$$
\eta_{\nu}\left(\phi_{\nu+1} X\right)=\eta_{\nu+2}(X)=-\eta_{\nu+1}\left(\phi_{\nu} X\right)
$$

Putting $X=\xi_{\nu}$ and $X=\xi_{\nu+1}$ into the first equation of Lemma 3.3 yields

Corollary 3.2 . We have

$$
\phi_{\nu+2} \xi_{\nu}=\xi_{\nu+1} \quad \text { and } \quad \phi_{\nu+2} \xi_{\nu+1}=-\xi_{\nu}
$$

We now investigate what the Kähler condition $\bar{\nabla} J=0$ tells us. First, using the Gauss formula we get

$$
\begin{aligned}
\bar{\nabla}_{X}(J Y) & =J \bar{\nabla}_{X} Y=J \nabla_{X} Y+g(A X, Y) J N \\
& =\phi \nabla_{X} Y+\eta\left(\nabla_{X} Y\right) N-g(A X, Y) \xi
\end{aligned}
$$

On the other hand, using the Weingarten formula, we obtain

$$
\begin{aligned}
\bar{\nabla}_{X}(J Y) & =\bar{\nabla}_{X}(\phi Y)+\bar{\nabla}_{X}(\eta(Y) N) \\
& =\nabla_{X}(\phi Y)-g(\phi A X, Y) N+(X \cdot \eta(Y)) N-\eta(Y) A X
\end{aligned}
$$

Comparing the tangential and normal components of the previous two equations leads to

LEMMA 3.4. For all $X, Y \in T M$ we have

$$
\left(\nabla_{X} \phi\right) Y=\eta(Y) A X-g(A X, Y) \xi
$$

and

$$
\left(\nabla_{X} \eta\right) Y=g(\phi A X, Y)
$$

From the second equation in Lemma 3.4 we derive

$$
g\left(\nabla_{X} \xi, Y\right)=X \cdot g(\xi, Y)-g\left(\xi, \nabla_{X} Y\right)=X \cdot \eta(Y)-\eta\left(\nabla_{X} Y\right)=g(\phi A X, Y)
$$

and hence

Corollary 3.3. For all $X \in T M$ we have

$$
\nabla_{X} \xi=\phi A X
$$

In addition to these equalities we have the well-known equations of Gauss and Codazzi. For us only the Codazzi equation will be relevant. Using the explicit expression for the Riemannian curvature tensor $\bar{R}$ of $G_{2}\left(\mathbb{C}^{m+2}\right)$ the Codazzi equation takes the following form. 
LEMma 3.5. For all $X, Y \in T M$ we have

$$
\begin{aligned}
\left(\nabla_{X} A\right) Y-\left(\nabla_{Y} A\right) X= & \eta(X) \phi Y-\eta(Y) \phi X-2 g(\phi X, Y) \xi \\
& +\sum_{\nu=1}^{3}\left\{\eta_{\nu}(X) \phi_{\nu} Y-\eta_{\nu}(Y) \phi_{\nu} X-2 g\left(\phi_{\nu} X, Y\right) \xi_{\nu}\right\} \\
& +\sum_{\nu=1}^{3}\left\{\eta_{\nu}(\phi X) \phi_{\nu} \phi Y-\eta_{\nu}(\phi Y) \phi_{\nu} \phi X\right\} \\
& +\sum_{\nu=1}^{3}\left\{\eta(X) \eta_{\nu}(\phi Y)-\eta(Y) \eta_{\nu}(\phi X)\right\} \xi_{\nu}
\end{aligned}
$$

\section{Proof of the Main Theorem}

In this section let us use the same notation as in Section 3. In order to prove our theorem we shall use some basic formulas derived from the Codazzi equation for a real hypersurface $M$ in $G_{2}\left(\mathbb{C}^{m+2}\right)$.

From the expression for the Riemannian curvature tensor $\bar{R}$ of $G_{2}\left(\mathbb{C}^{m+2}\right)$ we have derived the Codazzi equation given in Lemma 3.5. If the shape operator $A$ of $M$ is parallel, then the equation of Codazzi implies

$$
\begin{aligned}
0=\eta(X) \phi & Y-\eta(Y) \phi X-2 g(\phi X, Y) \xi \\
& +\sum_{\nu=1}^{3}\left\{\eta_{\nu}(X) \phi_{\nu} Y-\eta_{\nu}(Y) \phi_{\nu} X-2 g\left(\phi_{\nu} X, Y\right) \xi_{\nu}\right\} \\
& +\sum_{\nu=1}^{3}\left\{\eta_{\nu}(\phi X) \phi_{\nu} \phi Y-\eta_{\nu}(\phi Y) \phi_{\nu} \phi X\right\} \\
& +\sum_{\nu=1}^{3}\left\{\eta(X) \eta_{\nu}(\phi Y)-\eta(Y) \eta_{\nu}(\phi X)\right\} \xi_{\nu} .
\end{aligned}
$$

Putting $X=\xi$, then we have

$$
0=\phi Y+\sum_{\nu=1}^{3}\left\{\eta_{\nu}(\xi) \phi_{\nu} Y-\eta_{\nu}(Y) \phi_{\nu} \xi-2 g\left(\phi_{\nu} \xi, Y\right) \xi_{\nu}\right\}+\sum_{\nu=1}^{3} \eta_{\nu}(\phi Y) \xi_{\nu}
$$

From this, taking an inner product with $\xi$, we have

$$
3 \sum_{\nu=1}^{3} \eta_{\nu}(\xi) g\left(\phi_{\nu} Y, \xi\right)+\sum_{\nu=1}^{3} \eta_{\nu}(\phi Y) \eta_{\nu}(\xi)=0
$$


This gives

$$
\sum_{\nu=1}^{3} \eta_{\nu}(\xi) \eta_{\nu}(\phi Y)=0
$$

Here let us replace $Y$ by $\phi Y$ in (1.3). Then by Lemma 3.1 we have for any $Y \in \mathfrak{D}$

$$
\sum_{\nu} \eta_{\nu}(\xi)^{2} \eta(Y)=0
$$

where $\mathfrak{D}^{\perp}=\operatorname{Span}\left\{\xi_{1}, \xi_{2}, \xi_{3}\right\}$. From this we can consider the following two cases:

If $\eta(Y) \neq 0$ for some $Y \in \mathfrak{D}$, then $\eta_{\nu}(\xi)=0$ for any $\nu=1,2,3$. This gives $\xi \in \mathfrak{D}$, that is, $\xi$ is orthogonal to $\xi_{1}, \xi_{2}, \xi_{3}$.

If $\eta(Y)=0$ for any $Y \in \mathfrak{D}$, then $\xi \in \mathfrak{D}^{\perp}$. Thus we summarise this fact as follows:

Proposition 4.1. Let $M$ be a real hypersurface in $G_{2}\left(\mathbb{C}^{m+2}\right)$ satisfying $\nabla A=0$, then $\xi \in \mathfrak{D}$ or $\xi \in \mathfrak{D}^{\perp}$.

CASE I. Let $\xi \in \mathfrak{D}$. Now let us show that this case can not occur. Then the formula (4.1) with $X=\xi \in \mathfrak{D}$ implies

$$
0=\phi Y-\sum\left\{\eta_{\nu}(Y) \phi_{\nu} \xi+2 g\left(\phi_{\nu} \xi, Y\right) \xi_{\nu}\right\}+\sum \eta_{\nu}(\phi Y) \xi_{\nu}
$$

for any $Y \in T_{x} M$. From this, putting $Y=\xi_{\mu}$, we have

$$
\sum_{\nu=1}^{3} g\left(\phi_{\nu} \xi, \xi_{\mu}\right) \xi_{\nu}=0
$$

From this and together with taking an inner product (4.4) with $\xi_{\mu}$, we have

$$
0=\eta_{\mu}(\phi Y)-2 \sum_{\nu=1}^{3} g\left(\phi_{\nu} \xi, Y\right) \delta_{\nu \mu}+\eta_{\mu}(\phi Y)=4 \eta_{\mu}(\phi Y)
$$

So by putting $Y=\phi \xi_{\mu}, \eta_{\mu}\left(\phi^{2} \xi_{\mu}\right)=0$ implies a contradiction.

CASE II. Let $\xi \in \mathfrak{D}^{\perp}=\operatorname{Span}\left\{\xi_{1}, \xi_{2}, \xi_{3}\right\}$. Then there exists a quaternionic Kaehler structure $J_{1} \in \mathfrak{J}$ such that $J_{1} N=J N$.

Now let us put $\xi=\xi_{1}$. Then by Lemmas 3.1,3.2 and 3.3 and Corollaries 3.1 and 3.2 we know the following

$$
\phi \xi_{2}=\phi_{2} \xi=\phi_{2} \xi_{1}=-\xi_{3}, \quad \phi \xi_{3}=\phi_{3} \xi=\phi_{3} \xi_{1}=\xi_{2}
$$


Here let us put $\xi=\xi_{1}$. Bearing this in mind, taking an inner product (4.1) with $\xi$, we have

$$
\begin{aligned}
0=- & 2 g(\phi X, Y)+\left\{-2 g\left(\phi_{1} X, Y\right)+\eta_{2}(X) g\left(\phi_{2} Y, \xi\right)\right. \\
& \left.-\eta_{2}(Y) g\left(\phi_{2} X, \xi\right)+\eta_{3}(X) g\left(\phi_{3} Y, \xi\right)-\eta_{3}(Y) g\left(\phi_{3} X, \xi\right)\right\} \\
& +\eta_{2}(\phi X) \eta\left(\phi_{2} \phi Y\right)-\eta_{2}(\phi Y) \eta\left(\phi_{2} \phi X\right)+\eta_{3}(\phi X) \eta\left(\phi_{3} \phi Y\right) \\
& -\eta_{3}(\phi Y) \eta\left(\phi_{3} \phi X\right) .
\end{aligned}
$$

On the other hand, by using the formulas in (4.5) we know that

$$
\begin{aligned}
\eta_{2}(\phi X) & =g\left(\xi_{2}, \phi X\right)=-g\left(\phi \xi_{2}, X\right)=\eta_{3}(X), \\
\eta_{3}(\phi X) & =g\left(\xi_{3}, \phi X\right)=-g\left(\phi \xi_{3}, X\right)=-\eta_{2}(X), \\
\eta\left(\phi_{2} \phi Y\right) & =g\left(\xi, \phi_{2} \phi Y\right)=-g\left(\phi_{2} \xi_{1}, \phi Y\right)=-\eta_{2}(Y)
\end{aligned}
$$

and

$$
\eta\left(\phi_{3} \phi Y\right)=g\left(\xi, \phi_{3} \phi Y\right)=-g\left(\phi_{3} \xi_{1}, \phi Y\right)=-\eta_{3}(Y)
$$

Substituting these formulas into (4.6) and also using the formula given in (4.5), we have

$$
\begin{aligned}
& -2 g(\phi X, Y)+\left\{-2 g\left(\phi_{1} X, Y\right)+\eta_{2}(X) \eta_{2}(\phi Y)-\eta_{2}(Y) \eta_{2}(\phi X)\right\} \\
& \quad+\left\{\eta_{3}(X) \eta_{3}(\phi Y)-\eta_{3}(Y) \eta_{3}(\phi X)\right\}+2 \eta_{2}(X) \eta_{3}(Y)-2 \eta_{2}(Y) \eta_{3}(X)=0
\end{aligned}
$$

From this, putting $X=\xi_{2}$ and $Y=\xi_{3}$ and also using the formulas in (4.5), we have

$$
\eta_{2}\left(\xi_{2}\right) \eta_{2}\left(\phi \xi_{3}\right)-\eta_{3}\left(\xi_{3}\right) \eta_{3}\left(\phi \xi_{2}\right)+2 \eta_{2}\left(\xi_{2}\right) \eta_{3}\left(\xi_{3}\right)=\eta_{2}\left(\xi_{2}\right)^{2}+\eta_{3}\left(\xi_{3}\right)^{2}+2 \eta_{2}\left(\xi_{2}\right) \eta_{3}\left(\xi_{3}\right)=0
$$

This makes a contradiction. So we are able to assert the complete proof of our Main Theorem given in the introduction.

\section{REFERENCES}

[1] J. Berndt, 'Riemannian geometry of complex two-plane Grassmannians', Rend. Sem. Mat. Univ. Politec. Torino 55 (1997), 19-83.

[2] J. Berndt and Y.J. Suh, 'Real hypersurfaces in complex two-plane Grassmannians', Monatsh. Math. 127 (1999), 1-14.

[3] J. Berndt and Y.J. Suh, 'Isometric flows on real hypersurfaces in complex two-plane complex Grassmannians', Monatsh. Math. 137 (2002), 87-98.

[4] M. Kimura, 'Real hypersurfaces of a complex projective space', Bull. Austral. Math. Soc. 33 (1986), 383-387.

[5] M. Kimura and S. Maeda, 'On real hypersurfaces of a complex projective space II', Tsukuba J. Math. 15 (1991), 547-561. 
[6] J.D. Pérez, 'Real hypersurfaces of quaternionic projective space satisfying $\nabla_{U_{i}} A=0$ ', J. Geom. 49 (1994), 166-177.

[7] J.D. Pérez and Y.J. Suh, 'Real hypersurfaces of quaternionic projective space satisfying $\nabla U_{i} R=0 '$, Differential Geom. Appl. 7 (1997), 211-217.

\author{
Kyungpook National University \\ Department of Mathematics \\ Taegu 702-701 \\ Korea \\ e-mail: $\quad$ yjsuh@bh.knu.ac.kr
}

